\title{
New Physics search in the processes of the electroweak top quark production
}

\author{
Alexey Baskakov ${ }^{1}$, Edward Boos ${ }^{1}$, Vycheslav Bunichev ${ }^{1}$, Mikhail Dubinin ${ }^{1}$, Lev Dudko ${ }^{1}$, Igor \\ Myagkov $^{1}$, Maxim Perfilov ${ }^{1}$, and Georgy Vorotnikov ${ }^{1}$ \\ ${ }^{1}$ Skobeltsyn Institute of Nuclear Physics, MSU, 119992 Moscow, Russia
}

\begin{abstract}
The processes involving single top quark production have an electroweak nature with unique properties. They are very interesting from both theoretical and experimental points of view. The article contains a brief overview of possible "New Physics" manifestations in the single top quark production processes.
\end{abstract}

\section{Introduction}

In various aspects the top quark $(\mathrm{t})$ is a very unique object. The top quark is the heaviest elementary particle found so far with a mass slightly less than the mass of the gold nucleus. In comparison with other quarks the difference comes from two experimental facts, namely, a very large top quark mass compared to masses of all other quarks and very small mixing to quarks of the first and second generations. The value of the top quark mass measured is known now with a precision of $0.28 \%$ $M_{\mathrm{t}}=172.44 \pm 0.48 \mathrm{GeV}$ [1] being the most precisely known quark mass. Top Yukawa coupling $\lambda_{t}=2^{3 / 4} G_{F}^{1 / 2} m_{\mathrm{t}}$ is very close to unity. The quark mixing in the Standard Model (SM) is encoded in matrix elements of the Cabibbo-Kobayashi-Maskawa matrix. The matrix element $V_{\mathrm{tb}}$ is close to one while the elements $V_{\mathrm{ts}}$ and $V_{\mathrm{td}}$ are significantly smaller than one. These two experimental facts, large mass and small mixing, lead to the conclusion that in SM the top quark decays to W-boson and bquark with a probability close to $100 \%$. The width of the top quark being calculated in SM at the NLO level [2] is about $1.4 \mathrm{GeV}$. From one side, the top width is much smaller than its mass, and therefore the top quark is a narrow resonance. From the other side the top width is significantly larger than a typical QCD scale $\Lambda=200 \mathrm{MeV}$. As a result the top quark life time $\left(\tau_{\mathrm{t}} \approx 5 \times 10^{-25} \mathrm{~s}\right)$ as predicted by SM is much smaller than a typical time for formation of QCD bound states $\left(\tau_{\mathrm{QCD}} \approx 1 / \Lambda_{\mathrm{QCD}} \approx 3 \times 10^{-24} \mathrm{~s}\right)$. Therefore, the top quark decays long before it can hadronize, so top quark containing hadrons does not exist [3]. Thus the top quark provides a very clean source for fundamental information.

Since the top quark decays before hadronization its spin properties are not spoiled. Therefore the spin correlation in top production and decays is an interesting issue of the top quark physics.

Three mechanisms of the single top production are distinguished by the virtuality $Q_{\mathrm{W}}^{2}$ of the Wboson involved: $t$-channel $\left(Q_{\mathrm{W}}^{2}<0\right), s$-channel $\left(Q_{\mathrm{W}}^{2}>0\right)$, associated tW $\left(Q_{\mathrm{W}}^{2}=M_{\mathrm{W}}^{2}\right)$. The single top quark production at hadron colliders was considered for the first time in Ref. [4] and later in Ref. [5][15]. The authors of Ref. [12, 15] studied the most complete tree-level set of processes in the SM that contribute to the single top quark production. QCD NLO and NNLO corrections to various single top production processes have been calculated in several papers [16]-[29]. In particular, NLO corrections 
to kinematic distributions were presented [20]. The influence of NLO corrections not only to the production but also to the subsequent top quark decay has been studied in Ref. [22, 25]. Potentially important corrections at the threshold region have been resummed up to NNLL accuracy [27]. MonteCarlo (MC) analyses of the production processes of the single top quark allowing to extract it from main backgrounds were performed in Ref. [15, 19].

In the single top quark $t$-channel and $s$-channel processes in the SM top quark is produced through the left-handed current interaction. The production is very similar to the top decay process turned backward in time. For the polarized top decay, it is well-known that the charged lepton tends to point along the direction of top spin [30]. In the production process this is the direction of the initial $\overline{\mathrm{d}}$-quark for the $s$-channel, and the dominant direction of the final $\overline{\mathrm{d}}$-quark for the $t$-channel. Therefore, in the top quark rest frame there is strong correlation in the angle of produced lepton with respect to one of the above directions [31,32]: $\frac{1}{\sigma} \frac{d \sigma}{d \cos \theta_{\ell}^{*}}=\frac{1}{2}\left(1+\cos \theta_{\ell}^{*}\right)$. Spin properties in the tW production process are more involved. Here, in order to maximize spin correlations, one can find a kinematic region in which top quarks are produced with the polarization vector preferentially close to the direction of the charged lepton or the d,s-quark momentum from the associated $\mathrm{W}$ decay. In this kinematic region, the direction of the produced charged lepton or the d,s-quark should be as close as possible to the direction of the initial gluon beam in the top quark rest frame [32].

Due to unique properties physics of the single top quark will be an important part of research programs for all future hadron and lepton colliders and will include studies of top quark properties, various new physics channels, and kinematical characteristics of top quark events as significant backgrounds to a number of other processes.

Many details of theoretical studies and experimental analysis of single top production and decay properties could be found in a number of review papers Ref. [33]-[39].

\section{Manifestation of "New Physics" in single top quark production processes.}

Since the top is so heavy and point-like at the same time one might expect possible deviations from the SM predictions more likely occur in the top sector.

Single top processes are extremely interesting for different types of possible manifestations of "New Physics". In general, two main situations are possible depending on relations between characteristic collision energy and thresholds of possible new states. If the collision energy is smaller than the production threshold $E_{\text {collisions }}<E_{\text {threshold }}$ new states can not be produced directly and "New Physics" may manifest as deviations in production cross sections and kinematic distributions due to possible anomalous couplings or the interference of new resonances with the SM contribution below thresholds. In the case of single top, the anomalous couplings could be anomalous Wtb and/or FCNC couplings. If the collision energy is greater than the production threshold $E_{\text {collisions }}>E_{\text {threshold }}$ new states can be produced directly in the production or in the decay of the top quark. Such states are predicted in many extensions of the SM and could include additional heavy vector $\left(\mathrm{W}^{\prime}\right)$ or heavy scalar $\left(\mathrm{H}^{+}\right)$bosons decaing to top and bottom quarks or rather light not yet excluded charged scalars appearing in the top quark decay.

\section{Anomalous Wtb couplings}

Generic parametrisation of the anomalous couplings follows from the effective Lagrangian approach [40]. There are number of dimension 6 effective operators which preserve the SM gauge 
invariance $[41,42]$ and lead to modifications of Wtb vertex. The effective Wtb vertex has the following form [43]:

$$
\mathcal{L}=\frac{g}{\sqrt{2}} V_{\mathrm{tb}} \overline{\mathrm{b}} \gamma^{\mu}\left(f_{V}^{L} P_{L}+f_{V}^{R} P_{R}\right) \mathrm{tW}_{\mu}^{-}-\frac{g}{\sqrt{2}} \overline{\mathrm{b}} \frac{V_{\mathrm{tb}} \sigma^{\mu \nu} \partial_{\nu} \mathrm{W}_{\mu}^{-}}{M_{\mathrm{W}}}\left(f_{T}^{L} P_{L}+f_{T}^{R} P_{R}\right) \mathrm{t}+\text { h.c. }
$$

where $M_{W}$ is the mass of the $W$ boson, $V_{t b}$ is the Cabibbo-Kobayashi-Maskawa matrix element, $P_{\mathrm{L}, \mathrm{R}}=$ $\frac{1 \mp \gamma_{5}}{2}, \sigma_{\mu \nu}=\frac{i}{2}\left(\gamma_{\mu} \gamma_{v}-\gamma_{v} \gamma_{\mu}\right), g$ is the coupling constant of the weak interaction, the form factor $f_{V}^{L}$ $\left(f_{V}^{R}\right)$ represents the left-handed (right-handed) vector coupling, and $f_{T}^{L}\left(f_{T}^{R}\right)$ represents the left-handed (right-handed) tensor coupling. The anomalous couplings $f_{V}^{L}, f_{V}^{R}, f_{T}^{L}, f_{T}^{R}$ are related to the constants in front of the effective operators [41] in the following way:

$$
\left|f_{V}^{L}\right|=1+\left|C_{\phi q}^{(3,3+3)}\right| \frac{v^{2}}{V_{t b} \Lambda^{2}}, \quad\left|f_{V}^{R}\right|=\frac{1}{2}\left|C_{\phi \phi}^{33}\right| \frac{v^{2}}{V_{t b} \Lambda^{2}}, \quad\left|f_{T}^{L}\right|=\sqrt{2}\left|C_{d W}^{33}\right| \frac{v^{2}}{V_{t b} \Lambda^{2}}, \quad\left|f_{T}^{R}\right|=\sqrt{2}\left|C_{u W}^{33}\right| \frac{v^{2}}{V_{t b} \Lambda^{2}} .
$$

Note that the couplings $C$ are naturally of the order of unity. Therefore, one may expect the natural value of $f$ couplings of the order of $\frac{v^{2}}{\Lambda^{2}}$ which is about 0.05 or less. The theoretical estimations performed in Refs. [44-47] have shown the Tevatron and the LHC collider potentials showing the natural size of the parameters which could be achieved. The expected limits could not be much better because uncertainties are dominated by systematics. Expected bounds could be improved by a factor of $2 \div 3$ at a Linear Collider esspecially if the $e \gamma$-collision mode will be realised $[48,49]$.

In the single top processes the Wtb anomalous couplings contribute to the production and to the subsequent decay of produced top quark, and also change the total width of the top quark. The resulting dependence of the signal process on the anomalous parameters is more complicated than a simple polinomial structure. Such a dependence affects spin correlations between production and decay and can be exploited in experimental analysis. For example, anomalous operators are detectable in the $\mathrm{W}$ boson helicity fractions in the decay of top quarks [50, 51]. This property is used in many LHC measurements of $\mathrm{W}$ boson helicity fractions [52-54] The tightest limits coming from $\mathrm{W}$ boson helicity fractions are obtained by the ATLAS Collboration [52] at $7 \mathrm{TeV}:-0.20<f_{V}^{R}<0.23,-0.14<$ $f_{T}^{L}<0.11,-0.08<f_{T}^{R}<0.04$, and by the CMS Collaboration [55]: $-0.05<f_{T}^{R}<0.03$

The recent and most tight direct limits ${ }^{1}$ on the anomalous couplings $f_{V}^{R}$ and $f_{T}^{L}$ in Wtb vertex are achieved by the CMS Collaboration in the single top quark production process ( $p p$ collisions at $\sqrt{s}=7 \mathrm{TeV}$ ) [57]. New method of modelling of the anomalous interactions in this analysis is performed using auxillary vector fields [58]. The resulting CMS limits are: $\left|f_{V}^{L}\right|>0.90,\left|f_{V}^{R}\right|<0.34$, $\left|f_{T}^{L}\right|<0.09$.

The best direct limit on the $f_{T}^{R}$ coupling in Wtb vertex is obtained from combination of measurements in the single and the pair top quark production processes on the Tevatron by the D0 Collaboration [59]: $\left|f_{T}^{R}\right|<0.35$. There are also results for $f_{V}^{R}$ and $f_{T}^{L}$ couplings: $\left|f_{V}^{R}\right|<0.55,\left|f_{T}^{L}\right|<0.22$. Assuming the scale $\Lambda=1 \mathrm{TeV}$ these limits translate to the corresponding limits on the anomalous operators to be $\left|C_{\phi q}^{(3,3+3)}\right|<14.7,\left|C_{\phi \phi}^{33}\right|<18.0,\left|C_{d W}^{33}\right|<2.5$, and $\left|C_{u W}^{33}\right|<4.1$.

\subsection{FCNC top quark anomalous couplings}

FCNC top quark anomalous couplings can be probed in the production or in the rare decays of top quark [33]. In particular, tug and tcg FCNC couplings may affect the single top production rate,

\footnotetext{
${ }^{1}$ Indirect limits on the anomalous Wtb couplings follow from measurements of the $b \rightarrow s \gamma$ decays[56], however, the limits are obtained under the assumption that there are no other BSM contributions in the loop.
} 
what was exploited at the Tevatron and the LHC to set the corresponding limits. The gauge invariant effective Lagrangian describing the top FCNC interections has the following form:

$$
\mathcal{L}_{\mathrm{FCNC}}=\frac{1}{\Lambda}\left[\kappa_{\mathrm{tq}}^{\gamma, \mathrm{Z}} e \overline{\mathrm{t}} \sigma_{\mu \nu} \mathrm{q} F_{\gamma, \mathrm{Z}}^{\mu \nu}+\kappa_{\mathrm{tq}}^{g} g_{s} \overline{\mathrm{t}} \sigma^{\mu \nu} \frac{\lambda^{i}}{2} \mathrm{q} G_{\mu \nu}^{i}\right]+\text { h.c. }
$$

where $q=u$ or $c$, with $u, c$ and $t$ representing the quark fields; $\kappa_{\mathrm{tq}}^{\gamma, \mathrm{Z}}$ and $\kappa_{\mathrm{tq}}^{g}$ define the strength of couplings with the photon, $\mathrm{Z}$ and gluon, respectively; $g_{s}$ and $\lambda^{i}$ are the strong coupling constant and color matrices; $G_{\mu \nu}^{i}$ is the gauge field tensor of the gluon. From the above effective Lagrangian one easily obtains the partial rare decay widths: $\Gamma(\mathrm{t} \rightarrow \mathrm{qg})=\left(\frac{\kappa_{\mathrm{tq}}^{g}}{\Lambda}\right)^{2} \frac{8}{3} \alpha_{s} m_{\mathrm{t}}^{3}$. The partial width is directly proportional to the ratio $\left(\frac{\kappa_{\mathrm{tq}}^{g}}{\Lambda}\right)^{2}$, therefore various authors and collaborations present limits either in terms of constants $\kappa$ or in terms of the corresponding branching ratio. The representative diagrams contributing to various processes [60-62] of single top production due to presence of FCNC interactions are shown in Fig. 1. The model independent analysis based on the signal diagrams in
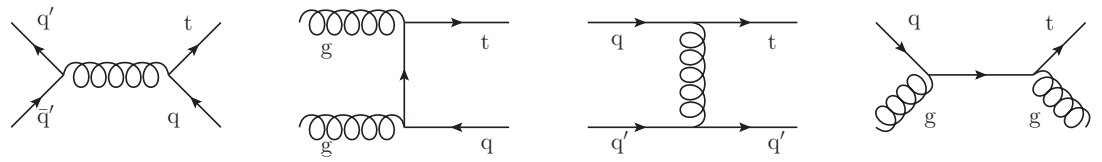

Figure 1. The representative leading order Feynman diagrams with FCNC tqg couplings.

Fig. 1 corresponds to the case when an extra hard jet is detected in association with the top quark. The most recent results on FCNC anomalous coupling limits in terms of both couplings and branchings are presented in Table 1. The accumulated luminosities for each of the analysis are listed explicitly in the table. One should stress the best current limits are far above very small SM value for the decay branching ratio $B R^{S M}(\mathrm{t} \rightarrow \mathrm{cg}) \approx 5 \times 10^{-11}$, however, being in the interesting range for some extensions of the SM [33].

Table 1. Observed 95\% C.L. upper limits on anomalous FCNC couplings and corresponding branching ratios.

\begin{tabular}{lcccc}
\hline & $\kappa_{t u}^{g} / \Lambda, \mathrm{TeV}^{-1}$ & $\kappa_{t c}^{g} / \Lambda, \mathrm{TeV}^{-1}$ & $B r(t \rightarrow u g)$ & $B r(t \rightarrow c g)$ \\
\hline D0 $\left(1.96 \mathrm{TeV}, 2.3 \mathrm{fb}^{-1}\right)[63]$ & $1.3 \times 10^{-2}$ & $5.7 \times 10^{-2}$ & $2.0 \times 10^{-4}$ & $3.9 \times 10^{-4}$ \\
\hline $\operatorname{CDF}\left(1.96 \mathrm{TeV}, 2.2 \mathrm{fb}^{-1}\right)[64]$ & & & $3.9 \times 10^{-4}$ & $5.7 \times 10^{-3}$ \\
\hline ATLAS $\left(7 \mathrm{TeV}, 2.05 \mathrm{fb}^{-1}\right)[65]$ & $6.9 \times 10^{-3}$ & $1.6 \times 10^{-2}$ & $5.7 \times 10^{-5}$ & $2.7 \times 10^{-4}$ \\
\hline ATLAS $\left(8 \mathrm{TeV}, 20 \mathrm{fb}^{-1}\right)[66]$ & $5.8 \times 10^{-3}$ & $1.3 \times 10^{-2}$ & $4.0 \times 10^{-5}$ & $1.7 \times 10^{-4}$ \\
\hline CMS $\left(7 \mathrm{TeV}, 5 \mathrm{fb}^{-1}\right)[57]$ & $1.8 \times 10^{-2}$ & $5.6 \times 10^{-2}$ & $3.6 \times 10^{-4}$ & $3.4 \times 10^{-3}$ \\
\hline \hline
\end{tabular}

\subsection{Top quark in the decay of new charged vector boson $\mathrm{W}^{\prime}$}

Single top could be produced in the decay of new charged vector $\left(\mathrm{W}^{\prime}\right)$ and observed as $s$-channel resonance decaying to the top and bottom quarks. Many extensions of the SM, in particular, composite models, modern variants of Technicolor models, superstrings inspired and Grand Unification models, models with extra dimensions, Left-Right symmetric models etc., predict an existence of such new vector boson (bosons) $\mathrm{W}^{\prime}$. In searches for $\mathrm{W}^{\prime}$ in a model independent way, the effective Lagrangian 
approach is used. Corresponding effective Lagrangians have the following form, neglecting higherdimensional structures:

$$
\mathcal{L}=\frac{V_{q_{i} q_{j}}}{2 \sqrt{2}} g_{w} \overline{\mathrm{q}}_{i} \gamma_{\mu}\left(a_{q_{i} q_{j}}^{R}\left(1+\gamma^{5}\right)+a_{q_{i} q_{j}}^{L}\left(1-\gamma^{5}\right)\right) \mathrm{W}^{\prime \mu} \mathrm{q}_{j}+\text { H.c. }
$$

where $a_{q_{i} q_{j}}^{R}, a_{q_{i} q_{j}}^{L}$ - left and right couplings of $\mathrm{W}^{\prime}$ to quarks, $g_{w}=e /\left(s_{w}\right)$ is the SM weak coupling constant and $V_{q_{i} q_{j}}$ is the SM CKM matrix element. The notations are taken such that for so-called SM-like $\mathrm{W}^{\prime} a_{q_{i} q_{j}}^{L}=1$ and $a_{q_{i} q_{j}}^{R}=0$. For the $\mathrm{W}^{\prime}$ searches the limits on $\mathrm{W}^{\prime}$ mass depend on both left and right couplings $a^{L}, a^{R}$, and for the case of right-handed interaction of $\mathrm{W}^{\prime}$ they depend on a relation between the $\mathrm{W}^{\prime}$ mass and the mass of possible right-handed neutrino $v_{\mathrm{R}}$. If the decay of $\mathrm{W}^{\prime}$ to the right-handed neutrino is kinematically allowed, the branching ratio of $\mathrm{W}^{\prime}$ decay to top and bottom quarks is smaller and corresponding mass limits are expected to be worse. The NLO corrections in the $s$-channel $\mathrm{W}^{\prime}$ production with subsequent decay were computed in Ref. [67] and an influence of $\mathrm{W}^{\prime}-\mathrm{W}^{\mathrm{SM}}$ interference was demonstrated in Ref. [68]. In the case when the $\mathrm{W}^{\prime}$ is a first $\mathrm{KK}$ state in models with extra deminsions, an additional interference with the rest of KK tower should be taken into account Ref. [69]. The 95\% C.L. limits obtained by the Tevatron at $\sqrt{s}=1.96 \mathrm{TeV}$ and the LHC collaborations at $\sqrt{s}=7,8$ and $13 \mathrm{TeV}$ are collected in the Table 2 for the cases when the couplings $a^{L}, a^{R}$ are equal to 1,0 (Left), 0,1 (Right) and 1,1 (Left, Right).

Table 2. Observed 95\% C.L. limits on the lowest bound of the mass of $\mathrm{W}^{\prime}$ boson.

\begin{tabular}{l|c|c|c|c}
\hline \hline Scenario & $M_{\mathrm{W}^{\prime}}($ Left $)$ & $\begin{array}{c}M_{\mathrm{W}^{\prime}}(\text { Right }), \\
M_{\mathrm{W}^{\prime}}>M_{\nu_{\mathrm{R}}}\end{array}$ & $\begin{array}{c}M_{\mathrm{W}^{\prime}} \text { (Right), } \\
M_{\mathrm{W}^{\prime}}<M_{\nu_{\mathrm{R}}}\end{array}$ & $\begin{array}{c}M_{\mathrm{W}^{\prime}}(\text { Left, Right }), \\
M_{\mathrm{W}^{\prime}}>M_{\nu_{\mathrm{R}}}\end{array}$ \\
\hline \hline D0 $(1.96 \mathrm{TeV})[70]$ & $863 \mathrm{GeV}$ & $885 \mathrm{GeV}$ & $890 \mathrm{GeV}$ & $916 \mathrm{GeV}$ \\
\hline CDF $(1.96 \mathrm{TeV})[71]$ & & $800 \mathrm{GeV}$ & $825 \mathrm{GeV}$ & \\
\hline CMS $(8 \mathrm{TeV})[72]$ & $1.92 \mathrm{TeV}$ & $2.15 \mathrm{TeV}$ & $2.02 \mathrm{TeV}$ & $2.15 \mathrm{TeV}$ \\
\hline ATLAS $(8 \mathrm{TeV})[73]$ & $1.70 \mathrm{TeV}$ & & $1.92 \mathrm{TeV}$ & \\
\hline \hline
\end{tabular}

\subsection{Charged Higgs boson and top quark}

Charged Higgs bosons appear if the Higgs sector includes two (or more) isodoublets. The most known is the two-Higgs-doublet model (THDM) [? ] and its special case, the effective Higgs potential of the Minimal Supersymmetric Standard Model (MSSM) [? ] with Yukawa sector of Type-II. Five physical scalar fields appear in the two-doublet models: two CP-even Higgs bosons h and H, a CP-odd Higgs boson $\mathrm{A}$ and two charged Higgs bosons $\mathrm{H}^{ \pm}$. If Yukawa sector of the model is Type-II, which is the case of MSSM, the couplings of the charged Higgs boson to u-type quarks are proportional to $\cot \beta$ and the couplings to the d-type quarks and charged leptons are proportinal to $\tan \beta\left(\tan \beta=v_{2} / v_{1}, \sqrt{v_{1}^{2}+v_{2}^{2}}=\right.$ $v=246 \mathrm{GeV})$. Charged Higgs interaction Lagrangian can be written as

$$
\mathcal{L}=\frac{g_{w} V_{q_{i} q_{j}}}{2 \sqrt{2}} \mathrm{H}^{+} \overline{\mathrm{q}}_{i}\left[g_{L}^{i j}\left(\frac{1-\gamma^{5}}{2}\right)+g_{R}^{i j}\left(\frac{1+\gamma^{5}}{2}\right)\right] \mathrm{q}_{\mathrm{j}}
$$

The LEP2 experiments defined a model-independent lower limit of about $80 \mathrm{GeV}$ on the charged Higgs mass [74] at a 95\% CL.

If the top quark can decay through the channel $\mathrm{t} \rightarrow \mathrm{H}^{+} \mathrm{b}$, so the mass of the charged Higgs boson is smaller than $m_{\mathrm{t}}-m_{\mathrm{b}}$, main single charged Higgs boson production mechanism is $\mathrm{t} \overline{\mathrm{t}} \rightarrow \mathrm{H}^{+} \mathrm{W}^{-} \mathrm{b} \overline{\mathrm{b}}$ 
when the top decays to $\mathrm{H}^{+} \overline{\mathrm{b}}$ and the antitop decays to $\mathrm{W}^{-} \mathrm{b}$. In the MSSM scenarios frequently considered [75] for moderate $\tan \beta$ the charged Higgs decays to $\tau^{+} v_{\tau}$. The ATLAS and CMS experiments determined an upper limits on $B R\left(\mathrm{t} \rightarrow \mathrm{H}^{+} \mathrm{b}\right)$ with the following decay $\mathrm{H}^{+} \rightarrow \tau^{+} v_{\tau}, \tau^{+}$ and $\mathrm{W}^{-}$decay hadronically. 95\% $\mathrm{CL}$ upper limits on $B R\left(\mathrm{t} \rightarrow \mathrm{H}^{+} \mathrm{b}\right) \times \mathrm{BR}\left(\mathrm{H}^{+} \rightarrow \tau^{+} v_{\tau}\right)$ in the mass range $m_{\mathrm{H}^{ \pm}}=80 \div 160 \mathrm{GeV}$ on the level $0.2 \div 1.2 \%$ have been set by ATLAS experiment [76] using $\sqrt{s}=8 \mathrm{TeV}$ data. For the e $\tau$ and $\mu \tau$ final states $95 \% \mathrm{CL}$ upper limit of $3 \div 9 \%$ for $B R\left(\mathrm{t} \rightarrow \mathrm{H}^{+} \mathrm{b}\right)$ have been found by ATLAS and CMS experiments in the mass range $m_{\mathrm{H}^{ \pm}}=80 \div 160 \mathrm{GeV}$ assuming $B R\left(\mathrm{H}^{+} \rightarrow \tau^{+} v_{\tau}\right)=1$ at the energy $\sqrt{s}=7 \mathrm{TeV}$ [77]. In the hadronic decay mode $\mathrm{H}^{+} \rightarrow \mathrm{cs}$ which is dominant for small $\tan \beta$ the upper limit on the level of $1 \div 5 \%$ (95\% CL) have been set by ATLAS experiment on $B R\left(\mathrm{t} \rightarrow \mathrm{H}^{+} \mathrm{b}\right)$ assuming that $B R\left(\mathrm{H}^{+} \rightarrow \mathrm{cs}\right)=1$ at the energy $\sqrt{s}=7 \mathrm{TeV}$ [78].

If the charged Higgs boson mass exceeds $m_{\mathrm{t}}-m_{\mathrm{b}}$, the main production mechanism is the bottomtop fusion (for the four-flavor scheme and for the five-flavor scheme). In the four-flavor scheme there are no b-quark PDF's causing a double-counting removal procedure [79] similar to the procedure for single top quark production. The contributions of the four- and the five-flavor schemes are calculated at NLO and combined using socalled "Santander matching scheme" [80]. In a number of popular MSSM scenarios the $\mathrm{H}^{+} \rightarrow \tau^{+} v_{\tau}$ mode dominates at moderate masses of $m_{\mathrm{H}^{ \pm}} \sim 200 \div 220 \mathrm{GeV}$. For large values of $m_{\mathrm{H}^{ \pm}}$and large $\tan \beta$ the channel $\mathrm{H}^{ \pm} \rightarrow$ tb becomes dominant. However, the contribution of $\mathrm{H}^{+} \rightarrow \tau^{+} v_{\tau}$ is non-negligible allowing one to set an upper limit of $0.004 \div 0.8 \mathrm{pb}$ on $\sigma\left(\mathrm{pp} \rightarrow \mathrm{t} \overline{\mathrm{b}} \mathrm{H}^{+}\right) \times \mathrm{BR}\left(\mathrm{H}^{+} \rightarrow \tau^{+} v_{\tau}\right)$ for $m_{\mathrm{H}^{ \pm}}=200 \div 1000 \mathrm{GeV}$ using ATLAS data at the energy $\sqrt{s}=8 \mathrm{TeV}$ [76]. First results of the CMS experiment on the direct charged Higgs boson production $\left(m_{\mathrm{H}^{ \pm}}>m_{\mathrm{t}}-m_{\mathrm{b}}\right)$ in the $\mathrm{H}^{+} \rightarrow \mathrm{tb}$ at the energy $\sqrt{s}=8 \mathrm{TeV}$ can be found in [81].

The available experimental limits for the charged scalar Higgs particle decaying to top and bottom quarks are published by D0 collaboration [82]. The mass limits are given in the mass range greater than $180 \mathrm{GeV}\left(m_{\mathrm{H}^{+}}>m_{\mathrm{t}}+m_{\mathrm{b}}\right)$ since lower mass range is covered with better precision from the top pair production with the top decay to the charged Higgs and b-quark. The limits strongly depend on the ratio of two Higgs vacuum expectation values $\tan \beta$ and the type of 2 HDMs (Type I, Type II and Type III).

\section{Conclusions and outlook}

The top quark, being the heaviest elementary particle discovered so far, has been observed first in the pair-production process and later in the single production mode. Single top quark registration at the Tevatron and then at the LHC is an important confirmation of the structure of the electroweak part of the Standard Model. The single top production cross sections measured at the Tevatron and the LHC are in a reasonable agreement with the SM computations including NLO and in several cases NNLO corrections. The single top production is a powerful tool to search for a delicate deviations from the SM. No such deviations have been observed yet but the limits on the anomalous Wtb and FCNC top quark couplings, as well as on the parameters of new vector and scalar bosons appearing in the top quark production or its subsequent decays have been reliably extracted. The mass limits for new resonances are expected to be much higher with increasing of accumulated statistics at nominal LHC energy and in future LHC runs with higher luminosity and energy. While the limits on anomalous couplings will be dominated by systematic uncertainties and therefore might be improved only by a factor of two-three, much tighter limits at a percentage level of accuracy could be achieved at a future Linear Collider. 


\section{Acknowledgments}

The authors are grateful to numerous colleagues from theory groups and numerous colleagues from the single top groups of the D0 and the CMS collaborations for clarifying discussions and joined studies. The work was supported by grant 16-12-10280 of the Russian Science Foundation.

\section{References}

[1] V. Khachatryan et al. [CMS Collaboration], Phys. Rev. D 93, no. 7, 072004 (2016)

[2] M. Jezabek and J. H. Kuhn, Phys. Rev. D 48, 1910 (1993) [Erratum-ibid. D 49, 4970 (1994)]; M. Jezabek and J. H. Kuhn, Nucl. Phys. B 314, 1 (1989).

[3] I. I. Bigi, Y. L. Dokshitzer, V. A. Khoze, J. H. Kuhn and P. M. Zerwas, Phys. Lett. B 181 (1986) 157.

[4] S. S. D. Willenbrock and D. A. Dicus, Phys. Rev. D 34, 155 (1986).

[5] C. P. Yuan, Phys. Rev. D 41, 42 (1990).

[6] G. V. Dzhikia and S. R. Slabospitsky, Sov. J. Nucl. Phys. 55, 1387 (1992); Phys. Lett. B 295, 136 (1992).

[7] S. Cortese and R. Petronzio, Phys. Lett. B 253, 494 (1991).

[8] R. K. Ellis and S. J. Parke, Phys. Rev. D 46, 3785 (1992).

[9] G. Bordes and B. van Eijk, Z. Phys. C 57, 81 (1993).

[10] D. O. Carlson, E. Malkawi, and C. P. Yuan, Phys. Lett. B 337, 145 (1994).

[11] T. Stelzer and S. Willenbrock, Phys. Lett. B 357, 125 (1995).

[12] A. P. Heinson, A. S. Belyaev, and E. E. Boos, Phys. Rev. D 56, 3114 (1997).

[13] S. Bar-Shalom, G. Eilam, A. Soni, and J. Wudka, Phys. Rev. D 57, 2957 (1998).

[14] T. Tait and C. P. Yuan, hep-ph/9710372.

[15] A. S. Belyaev, E. E. Boos, and L. V. Dudko, Phys. Rev. D 59, 075001 (1999)

[16] G. Bordes and B. van Eijk, Nucl. Phys. B 435, 23 (1995)

[17] R. Pittau, Phys. Lett. B 386, 397 (1996)

[18] M. C. Smith and S. Willenbrock, Phys. Rev. D 54, 6696 (1996); T. Stelzer, Z. Sullivan, and S. Willenbrock, Phys. Rev. D 56, 5919 (1997);

[19] T. Stelzer, Z. Sullivan, and S. Willenbrock, Phys. Rev. D 58, 094021 (1998).

[20] B. W. Harris et al., Phys. Rev. D 66, 054024 (2002).

[21] Z. Sullivan, Phys. Rev. D 70, 114012 (2004).

[22] J. Campbell, R. K. Ellis, and F. Tramontano, Phys. Rev. D 70, 094012 (2004).

[23] J. M. Campbell and F. Tramontano, Nucl. Phys. B 726, 109 (2005); J. M. Campbell, R. Frederix, F. Maltoni and F. Tramontano, Phys. Rev. Lett. 102, 182003 (2009).

[24] Q. H. Cao, R. Schwienhorst, and C. P. Yuan, Phys. Rev. D 71, 054023 (2005); Q. -H. Cao, R. Schwienhorst, J. A. Benitez, R. Brock, C. -P. Yuan, Phys. Rev. D72, 094027 (2005); S. Heim, Q. -H. Cao, R. Schwienhorst, C. -P. Yuan, Phys. Rev. D81, 034005 (2010);

[25] R. Schwienhorst, C. -P. Yuan, C. Mueller and Q. -H. Cao, Phys. Rev. D 83, 034019 (2011).

[26] N. Kidonakis, Phys. Rev. D 74, 114012 (2006); N. Kidonakis, Phys. Rev. D 75, 071501 (2007); N. Kidonakis, Nucl. Phys. A 827, 448C (2009); N. Kidonakis, Phys. Rev. Lett. 102, 232003 (2009); N. Kidonakis, Phys. Rev. D 81, 054028 (2010); N. Kidonakis, Phys. Rev. D 82, 054018 (2010); N. Kidonakis, Phys. Rev. D 83, 091503 (2011);

[27] N. Kidonakis, Phys. Part. Nucl. 45, no. 4, 714 (2014).

[28] M. Brucherseifer, F. Caola and K. Melnikov, Phys. Lett. B 736, 58 (2014) 
[29] E. L. Berger, J. Gao, C.-P. Yuan and H. X. Zhu, arXiv:1606.08463 [hep-ph].

[30] M. Jezabek and J. H. Kuhn, Phys. Lett. B 329, 317 (1994).

[31] G. Mahlon and S. J. Parke, Phys. Rev. D 55, 7249 (1997); G. Mahlon and S. J. Parke, Phys. Lett. B 476, 323 (2000).

[32] E. E. Boos and A. V. Sherstnev, Phys. Lett. B 534, 97 (2002).

[33] M. Beneke et al., "Top quark physics," arXiv:hep-ph/0003033.

[34] T. M. P. Tait and C. P. P. Yuan, Phys. Rev. D 63, 014018 (2000)

[35] S. Willenbrock, hep-ph/0211067.

[36] R. Kehoe, M. Narain and A. Kumar, Int. J. Mod. Phys. A 23, 353 (2008)

[37] A. Heinson and T. R. Junk, Ann. Rev. Nucl. Part. Sci. 61, 171 (2011)

[38] E. Boos and L. Dudko, Int. J. Mod. Phys. A 27, 1230026 (2012)

[39] E. Boos, O. Brandt, D. Denisov, S. Denisov and P. Grannis, Phys. Usp. 58, no. 12, 1133 (2015)

[40] W. Buchmuller and D. Wyler, Nucl. Phys. B 268, 621 (1986).

[41] J. A. Aguilar-Saavedra, Nucl. Phys. B 812 (2009) 181

[42] C. Zhang, N. Greiner, and S. Willenbrock, arXiv:1201.6670 [hep-ph]; C. Zhang and S. Willenbrock, Phys. Rev. D 83, 034006 (2011).

[43] G. L. Kane, G. A. Ladinsky and C. P. Yuan, Phys. Rev. D 45, 124 (1992).

[44] E. Boos, L. Dudko, and T. Ohl, Eur. Phys. J. C 11, 473 (1999)

[45] S. Tsuno, I. Nakano, Y. Sumino and R. Tanaka, Phys. Rev. D 73, 054011 (2006)

[46] J. A. Aguilar-Saavedra, Nucl. Phys. B 804, 160 (2008)

[47] W. Bernreuther, P. Gonzalez and M. Wiebusch, Eur. Phys. J. C 60, 197 (2009)

[48] E. Boos, M. Dubinin, A. Pukhov, M. Sachwitz, H. J. Schreiber, Eur. Phys. J. C21, 81-91 (2001).

[49] E. Boos, A. Pukhov, M. Sachwitz and H. J. Schreiber, Phys. Lett. B 404, 119 (1997)

[50] J. A. Aguilar-Saavedra, J. Carvalho, N. F. Castro, F. Veloso and A. Onofre, Eur. Phys. J. C 50 (2007) 519

[51] C. -R. Chen, F. Larios and C. -P. Yuan, Phys. Lett. B 631, 126 (2005) [AIP Conf. Proc. 792, 591 (2005)]

[52] G. Aad et al. [ATLAS Collaboration], JHEP 1206 (2012) 088

[53] V. Khachatryan et al. [CMS Collaboration], JHEP 1501 (2015) 053

[54] [CMS Collaboration], CMS-PAS-TOP-12-025.

[55] S. Chatrchyan et al. [CMS Collaboration], JHEP 1310 (2013) 167

[56] J. Drobnak, S. Fajfer and J. F. Kamenik, Nucl. Phys. B 855, 82 (2012);

[57] CMS Collaboration [CMS Collaboration], CMS-PAS-TOP-14-007.

[58] E. Boos, V. Bunichev, L. Dudko and M. Perfilov, arXiv:1607.00505 [hep-ph].

[59] V. M. Abazov et al. [D0 Collaboration], Phys. Lett. B 713, 165 (2012)

[60] T. Han, M. Hosch, K. Whisnant, B. -L. Young and X. Zhang, Phys. Rev. D 58, 073008 (1998)

[61] J. J. Liu, C. S. Li, L. L. Yang and L. G. Jin, Phys. Rev. D 72, 074018 (2005)

[62] J. Gao, C. S. Li, J. J. Zhang, H. X. Zhu, Phys. Rev. D80, 114017 (2009);

[63] V. M. Abazov et al. [D0 Collaboration], Phys. Lett. B 693, 81 (2010)

[64] T. Aaltonen et al. [CDF Collaboration], Phys. Rev. Lett. 102, 151801 (2009)

[65] G. Aad et al. [ATLAS Collaboration], Phys. Lett. B 712, 351 (2012)

[66] G. Aad et al. [ATLAS Collaboration], Eur. Phys. J. C 76, no. 2, 55 (2016)

[67] Z. Sullivan, Phys. Rev. D 66, 075011 (2002)

[68] E. Boos, V. Bunichev, L. Dudko and M. Perfilov, Phys. Lett. B 655, 245 (2007) 
[69] E. E. Boos, I. P. Volobuev, M. A. Perfilov and M. N. Smolyakov, Theor. Math. Phys. 170, 90 (2012)

[70] V. M. Abazov et al. [D0 Collaboration], Phys. Lett. B 699, 145 (2011)

[71] T. Aaltonen et al. [CDF Collaboration], Phys. Rev. Lett. 103, 041801 (2009)

[72] V. Khachatryan et al. [CMS Collaboration], JHEP 1602, 122 (2016)

[73] G. Aad et al. [ATLAS Collaboration], Phys. Lett. B 743 (2015) 235

[74] ALEPH Collaboration, Phys. Lett. B 543, 1 (2002); DELPHI Collaboration, Eur. Phys. J. C 34, 399 (2004); L3 Collaboration, Phys. Lett. B 575, 208 (2003); OPAL Collaboration, Eur. Phys. J. C 72, 2076 (2012).

[75] S. Heinemeyer, W. Hollik, and G. Weiglein, Comput. Phys. Commun. 124, 76 (2000)

[76] ATLAS Collaboration, JHEP 03, 088 (2015)

[77] ATLAS Collaboration, JHEP 03, 076 (2013); CMS Collaboration, JHEP 07, 143 (2012).

[78] ATLAS Collaboration, Eur. Phys. J. C 73, 2465 (2013).

[79] LHC Higgs Cross Section Working Group Collaboration, arXiv:1307.1347[hep-ph]

[80] R. Harlander, M. Kramer, and M.Schumacher, CERN Technical Report PH-TH/2011-134, 2011 (arXiv:1112.3478[hep-ph])

[81] CMS Collaboration, JHEP 1511, 018 (2015)

[82] V. M. Abazov et al. [D0 Collaboration], Phys. Rev. Lett. 102, 191802 (2009) 